\title{
Is the Sherrington-Kirkpatrick Model relevant for real spin glasses?
}

\author{
A. P. Young \\ Department of Physics, University of California, Santa Cruz, California 95064 \\ E-mail: peter@physics.ucsc.edu
}

\begin{abstract}
I describe the results of numerical simulations which test whether the SherringtonKirkpatrick model, which was solved by Parisi, applies to short-range spin glasses. I conclude that it probably does for dimension $d$ greater than 6 , but there appear to be some differences in lower dimensions. In particular, there does not appear to be the line of transitions in a magnetic field (AT line) which is seen in the SK model.
\end{abstract}

\section{Introduction}

It is a pleasure to give this talk at a meeting honoring the scientific contributions of David Sherrington. I've know David for most of his career and all of mine, since he was the external examiner for my D. Phil. viva in 1973. Subsequently we were colleagues at Imperial College for several years, and David hosted my sabbatical in Oxford in 2001-02. In addition, we have had many discussions on spin glasses over the years.

Spin glasses are systems with two key ingredients: disorder, and frustration, i.e. competition between different terms in the Hamiltonian so they can not all be satisfied simultaneously. Figure 1 shows a toy example of frustration with a single square of Ising spins. (Ising spins can only point up or down.) The "+" or "-" on the bonds indicates a ferromagnetic or antiferromagnetic

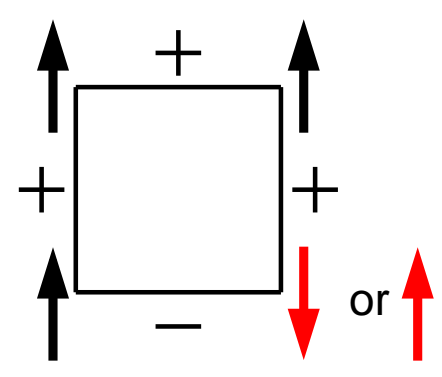

Figure 1. A Toy model which shows frustration. If the interaction on the bond is a "+", the spins want to be parallel and if it is a "-" they want to be antiparallel. Clearly all these conditions can not be met simultaneously so there is competition or "frustration". 
interaction respectively. In this example, with one negative bond, it is impossible to minimize the energy of all the bonds so there is competition or "frustration".

Most theoretical work therefore uses the simplest model with the features of disorder and frustration, the Edwards Anderson [1] (EA) model, whose Hamiltonian is

$$
\mathcal{H}=-\sum_{\langle i, j\rangle} J_{i j} S_{i} S_{j}-\sum_{i} h_{i} S_{i}
$$

The Ising spins $S_{i}$ take values \pm 1 and lie on the sites $i$ of a three-dimensional, simple cubic lattice with $N=L^{3}$ sites. Periodic boundary conditions are applied. The interactions $J_{i j}$ are between nearest neighbors and are independent random variables with a symmetric distribution and and standard deviation unity, i.e.

$$
\left[J_{i j}\right]_{\mathrm{av}} \equiv J_{0}=0 ; \quad\left[J_{i j}^{2}\right]_{\mathrm{av}}^{1 / 2} \equiv J=1,
$$

where $[\cdots]_{\text {av }}$ means an average over the disorder. We will also use the notation $\langle\cdots\rangle$ to indicate a thermal average for a particular set of interactions. The precise form of the distribution of the $J_{i j}$ is not very important but, for technical reasons, it will be convenient to take a Gaussian distribution in the simulations reported here. In some of what follows we shall also include a magnetic field $h_{i}$.

Motivated by the pioneering work of Edwards and Anderson [1] (EA), David, together with Scott Kirkpatrick, [2] (SK) proposed that the mean field theory of spin glasses should be the exact solution of an infinite-range version of the EA model in which there is no lattice and the distribution of the $J_{i j}$ is the same for all distinct pairs $i$ and $j$. In order to get a sensible thermodynamic limit, one has to scale the variance of the distribution by $N$, i.e. $\left[J_{i j}^{2}\right]_{\text {av }}^{1 / 2}=1 / \sqrt{N}$.

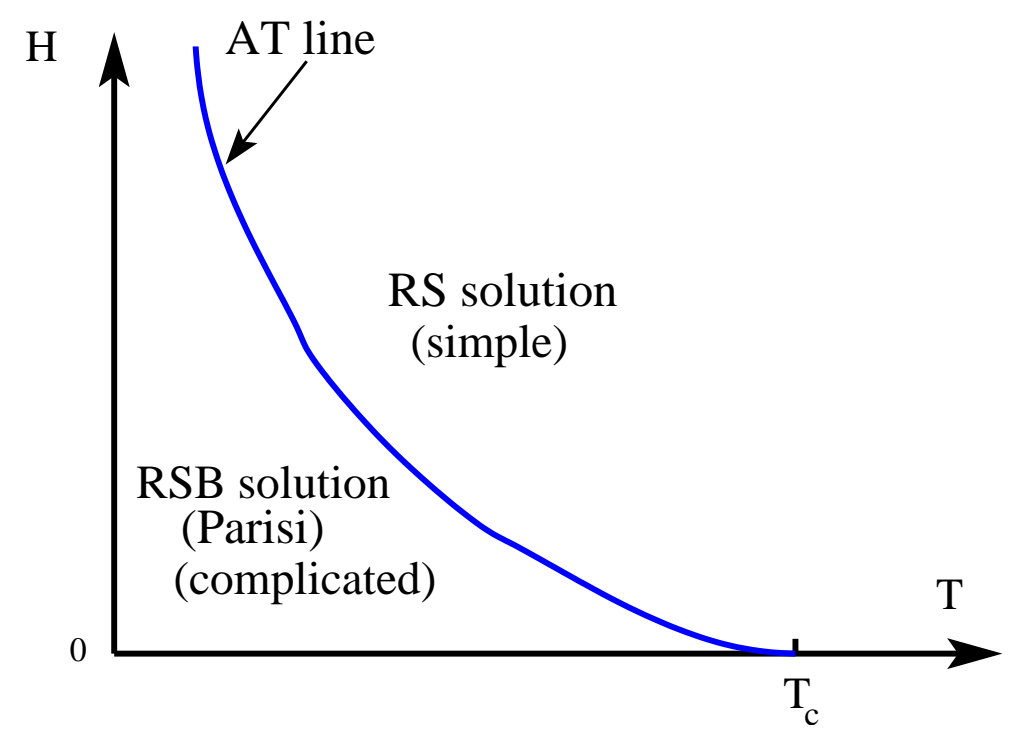

Figure 2. The magnetic field-temperature phase diagram of the SK model according to Almeida and Thouless [3]. Above the line, the replica symmetric solution of SK is correct, while below the line one has to break the symmetry in a more complicated way, i.e. the "replica symmetry breaking" (RSB) solution of Parisi.

The SK solution of their model was of the same type as that found by EA, what we now call the "replica symmetric" (RS) solution. Perhaps the most important advance in the SK paper, 


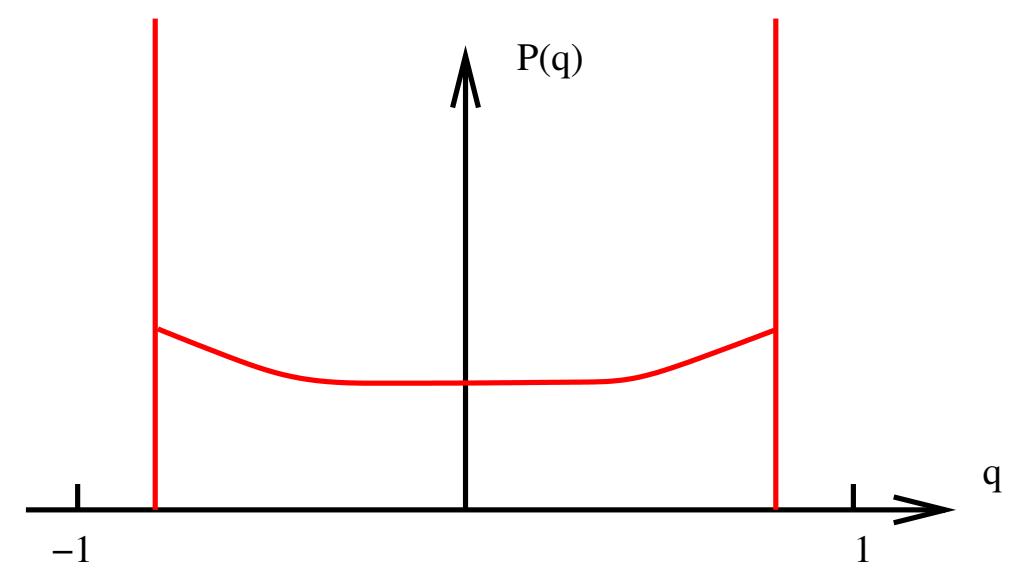

Figure 3. The order parameter distribution in the Parisi field for $h=0$. Note that it is symmetric because of time-reversal symmetry. There is a delta function at $q=q_{\text {EA }}$ where the two copies are in the same valley (and one at $-q_{\mathrm{EA}}$ where one copy is the time-reversed image of the other). There is also a "non-trivial" part with a non-zero weight even at $q=0$, where the two copies are in different valleys.

relative to EA, is that SK realized that the $R S$ solution can not be correct since the entropy went negative at low temperatures (which is impossible for an Ising model since the states can be counted).

Almeida and Thouless [3] subsequently clarified what was going wrong. They looked at the stability of the RS solution in the magnetic field-temperature plane. They found that the RS solution was stable above a line in this plane, see Fig. 2, but was unstable below this line.

The theory used the "replica trick" in which averages over the quenched disorder are carried out by making $n$ copies ("replicas") of the system and letting $n$ tend to zero. In the RS solution, all pairs of replicas are equivalent and there is a single order parameter. It was clear that this "replica symmetry" had to be broken below the AT line but how to do so was not obvious, since the size of the space, $n$, is non-integer and tends to zero. In a tour-de-force, Parisi $[4,5]$ proposed a hierarchical "replica symmetry breaking" (RSB) solution with an infinite number of parameters. Twenty seven years after Parisi introduced his solution, its free energy was rigorously proved to be exact by Tallegrand [6].

The Parisi solution has two important features.

- The first, as we have seen, is that there is line of phase transitions in a magnetic field (AT line).

- The second is that below the AT line the order parameter is not just a single number but a function (actually a probability distribution), i.e. there are an infinite number of order parameters. The theory involves the overlap between two spin configurations, $q$, defined by

$$
q=\frac{1}{N} \sum_{i=1}^{N} S_{i}^{(1)} S_{i}^{(2)},
$$

where (1) and (2) refer to two (real) copies of the system with the same interactions. Below the AT line, $q$ has a non-trivial distribution $P(q)$, which, for zero field, looks as in Fig. 3 .

As soon as the field becomes non-zero the negative- $q$ region is eliminated because the symmetry is now broken (one of the two states which were related by time-reversal symmetry in zero field is suppressed), and there is a non-zero weight in the distribution between $q_{\mathrm{EA}}$ 
(which turns out to be only very weakly dependent on field) and $q_{\min }$ where $0<q_{\min }<q_{\mathrm{EA}}$. As the AT line is approached from below $q_{\text {min }}$ tends to $q_{\mathrm{EA}}$, and above the AT line the distribution is just a delta function at $q_{\mathrm{EA}}$. This is the replica symmetric solution.

The motivations for SK to introduce their infinite-range model were (i) it should be exactly solvable, and (ii) the behavior of real spin glasses might have similar features to the exact solution. We have seen that (i) is correct, though the exact solution was harder to find than expected. Whether (ii) is a correct has been, and continues to be, controversial.

The hypothesis that real spin glasses behave in a similar way to the SK model is called the RSB scenario for the spin glass state. Another scenario, with different predictions, has also been proposed called the "droplet picture". This was developed in most detail by Fisher and Huse $[7,8,9]$, though similar ideas were also proposed by Bray and Moore [10] and McMillan [11]. The droplet picture differs from the RSB scenario in two important respects: (i) there is no AT line, and (ii) the order parameter is just a single number $q_{\mathrm{EA}}$, since the distribution $P(q)$ is just two delta functions (in zero field) at $q= \pm q_{\mathrm{EA}}$, i.e. the continuous "non-trivial" part in Fig. 3 is missing.

In the rest of this presentation, I will use numerical simulations to ascertain whether the $\mathrm{RSB}$ or droplet picture (or neither) is correct. In Sec. 2, I discuss the finite-size scaling method used to analyze the data, and in Sec. 3 I describe the parallel tempering Monte Carlo method which speeds up equilibration at low temperature. In addition to the short-range EA model in three-dimensions described above, it will also be useful to consider a one-dimensional model with long-range interactions. This is discussed in Sec. 4. Results for the order parameter distribution are discussed in Sec. 5 and results concerning the existence of an AT line are discussed in Sec. 6. Finally, the conclusions are summarized in Sec. 7.

\section{Finite-Size Scaling}

In the later sections we will use numerical simulations to investigate phase transitions in spin glasses. Of course, a sharp transition can only occur in the thermodynamic limit, whereas simulations are carried out on finite-size lattices. We therefore use the technique of finite-size scaling (FSS) to extrapolate from results on a range of finite sizes to the thermodynamic limit. Following Ballesteros et al. [12], we shall find the correlation length of the finite system to be a particularly useful quantity to analyze by FSS.

To extract a correlation length we define the spin glass susceptibility at finite wavevector k:

$$
\chi_{S G}(\mathbf{k})=\frac{1}{N} \sum_{i, j}\left[\left\langle S_{i} S_{j}\right\rangle^{2}\right]_{\mathrm{av}} e^{i \mathbf{k} \cdot\left(\mathbf{R}_{i}-\mathbf{R}_{j}\right)} .
$$

We then determine the finite-size spin glass correlation length $\xi_{L}$ from the Ornstein-Zernicke equation:

$$
\chi_{S G}(\mathbf{k})=\frac{\chi_{S G}(\mathbf{0})}{1+\xi_{L}^{2} \mathbf{k}^{2}+\ldots}
$$

by fitting to $\mathbf{k}=0$ and $\mathbf{k}=\mathbf{k}_{\min }=\frac{2 \pi}{L}(1,0,0)$.

The basic assumption in FSS is that the size dependence of the results comes from the ratio $L / \xi_{\text {bulk }}$ where

$$
\xi_{\text {bulk }} \sim\left(T-T_{S G}\right)^{-\nu}
$$

is the bulk (i.e. infinite-system size) correlation length. In particular, the correlation length $\xi_{L}$ of the simulated system, which has linear size $L$, varies as

$$
\frac{\xi_{L}}{L}=X\left(L^{1 / \nu}\left(T-T_{S G}\right)\right) .
$$


Since $\xi_{L} / L$ is dimensionless it turns out that there is no power of $L$ multiplying the scaling function $X$. This is very useful because, according to Eq. (7), data for different sizes intersect at $T_{S G}$. Hence the transition temperatures can be determined by eye. Furthermore, if there is long-range spin glass order then the data should splay out below $T_{S G}$. This works very well for the Ising spin glass $[12,13]$

If we had used $\chi_{S G}$, rather than $\xi_{L}$ as the quantity to analyze, there would have been an additional factor of $L$ to an unknown power multiplying the scaling function in Eq. (7) which would have complicated the analysis,

\section{Parallel Tempering}

Experimentally, spin glass dynamics becomes very slow at low temperatures, because the system has a very complicated "energy landscape" with many "valleys", separated by barriers, in which the system gets trapped. In Monte Carlo simulations too, the system gets trapped in a valley at low temperature, and conventional Monte Carlo simulations take a huge amount of time to equilibrate in this region. Most Monte Carlo simulations of spin glasses, including those described here, therefore use a modified approach known as parallel tempering (replica exchange) [14], which helps the system get over barriers and equilibrate at low temperature. This approach, which I now describe, is of quite general applicability to problems with a complicated energy landscape.

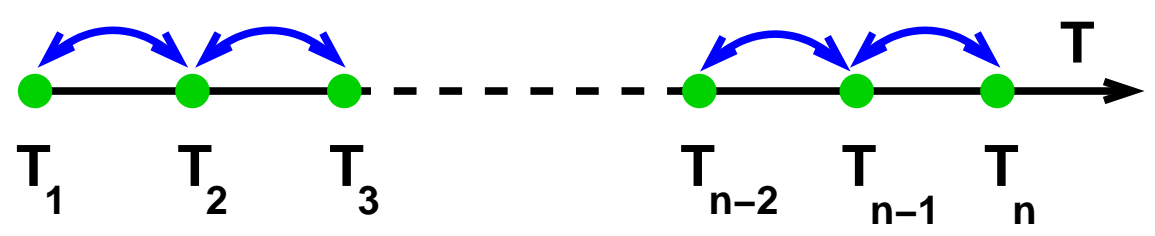

Figure 4. Sketch illustrating the temperatures swaps in the parallel tempering method.

One simulates a copy of the system, with the same interactions, at each of $n$ temperatures between $T_{\min }=T_{1}$ and $T_{\max }=T_{n}$, see Fig. 4 . The highest temperature is chosen so that the system equilibrates fast; it has enough energy to easily get over the barriers. The lowest temperature is chosen in the region one wants to study. In addition to the usual single-spin updates at each temperature, one also performs temperature swaps in which the entire spin configurations at neighboring temperatures, $T_{l}$ and $T_{l+1}$, are swapped with probability

$$
P_{\text {swap }}=\exp \left[\left(\beta_{l}-\beta_{l+1}\right)\left(E_{l}-E_{l+1}\right)\right] .
$$

where $\beta_{l}=1 / T_{l}$, and $E_{l}$ is the total energy of the copy at temperature $T_{l}$. It is not difficult to check that Eq. (8) satisfies the detailed balance condition, and so eventually the whole set of copies will come to thermal equilibrium.

The price one pays is that one has to simulate several (sometimes many) copies of the system. Not all this is wasted, though, since one usually does want results at several temperatures. However, there is an overhead because one ends up doing more sweeps than necessary at high temperatures, and quite often one is forced to use more temperatures than are really needed. This is particularly the case for large sizes because the spacing between temperatures has to vary with $N$ as $N^{-1 / 2}$, so the number of temperatures varies as $N^{1 / 2}$, in order that the probability in Eq. (8) is significant. Physically this is because the same spin configuration must one which occurs with significant probability at both $T_{l}$ and $T_{l+1}$. However, at low temperature, one finds that the speed up of the relaxation time more than compensates for the overhead in simulating a large number of temperatures. 


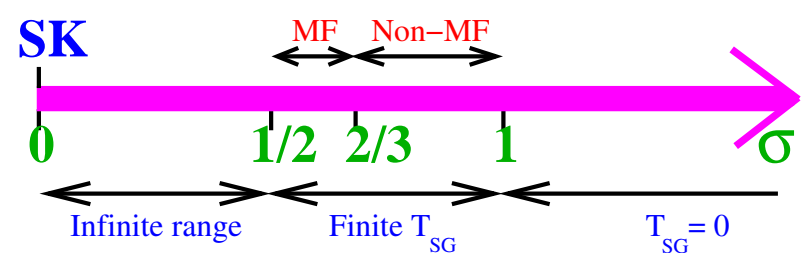

Figure 5. The different types of behavior of the long-range model in $d=1$ as a function of the power $\sigma$ with which the interactions fall off with distance.

\section{Model with long-range interactions}

For short-range models, there is a lower critical dimension $d_{l}$ (equal to [15] about 2.5), below which the finite-temperature zero-field transition disappears, and an upper critical dimension $d_{l}$ (equal to 6) where the zero field transition is described by mean field exponents, i.e. those of the EA-SK theory.

It is therefore of interest to investigate spin glasses not only in three dimensions but also in higher dimensions. Even with parallel tempering it is difficult to equilibrate more than of order a thousand spins at low temperatures, and since $N=L^{d}$ the range of $L$ is very limited at high $d$. We will therefore also consider a one-dimensional model in which the interactions fall of with a power of the distance:

$$
J_{i j} \propto \frac{\epsilon_{i j}}{r_{i j}^{\sigma}}, \quad\left[\epsilon_{i j}\right]_{\mathrm{av}}=0, \quad\left[\epsilon_{i j}^{2}\right]_{\mathrm{av}}=1
$$

For each $\sigma$ we rescale the interactions so that the mean field transition temperature $T_{S G}^{M F}$ is unity (for zero field).

This model is useful because one can make an analogy between varying $d$ for short-range models, and varying $\sigma$ for the long-range model in $d=1$. This has been well established for ferromagnets, e.g. [16]. In particular there is a $\sigma_{l}$ above which $T_{S G}=0$, and a $\sigma_{u}$ below which the zero-field critical behavior is mean-field like, where $\sigma_{l}=1[9]$ and $\sigma_{u}=2 / 3$ [17]. Note too that $\sigma=0$ is SK model and the range $0 \leq \sigma \leq 1 / 2$ is infinite-range since $\sum_{j}\left[J_{i j}^{2}\right]_{\text {av }}$ diverges. Furthermore, $\sigma \rightarrow \infty$ is the nearest-neighbor model. Figure 5 summarizes the behavior of the $d=1$ long-range model as a function of $\sigma$

\section{Results for the order parameter distribution $\mathbf{P}(\mathbf{q})$}

In this section we consider only zero magnetic field.

As discussed in Sec. 1, the RSB picture of the spin glass state below $T_{S G}$ predicts that the order parameter distribution $P(q)$ should have a continuous part with a finite weight even at $q=0$. By contrast the droplet theory predicts that the weight in the continuous part should be zero in the thermodynamic limit. More precisely the prediction is [7] that $P(0) \propto 1 / L^{\theta}$ where $\theta(>0)$ is a stiffness exponent, estimated to be about $0.20[20,21]$ for the Ising spin glass in three dimensions.

The left hand side of Fig. 6, from Ref. [18], shows that $P(0)$ is independent of size for $3 \leq L \leq 8$ at at temperature of 0.20 , far below the transition temperature of about 0.95 . The right hand side of this figure shows similar data, from Ref. [19], for the long-range model with $\sigma=0.75$, in the region predicted to have a finite transition temperature with non mean-field exponents. The temperature is 0.10 compared with the transition temperature of about 0.62 . The stiffness exponent is expected to be [9] $d-\sigma=0.25$. For a large range of sizes, $32 \leq L \leq 512$, one again finds that $P(0)$ is independent of size. 

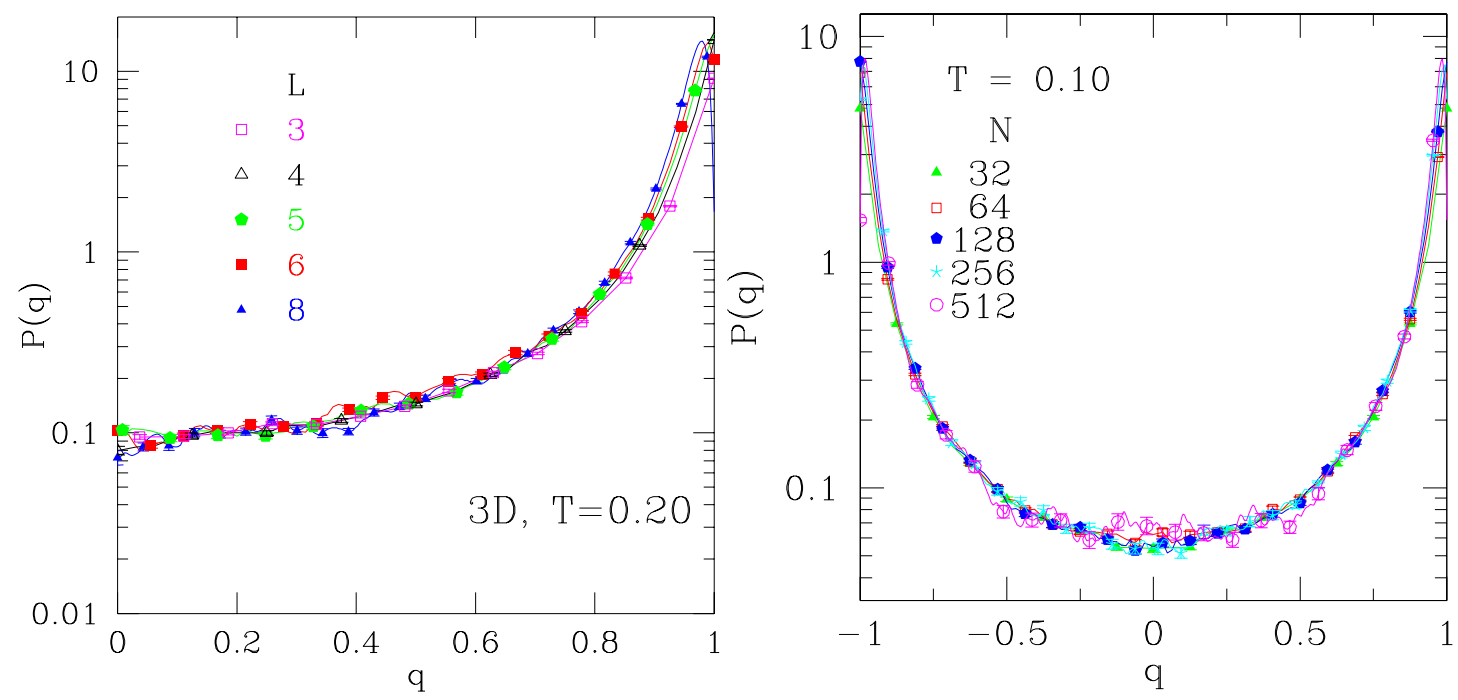

Figure 6. The left hand figure is the order parameter distribution in zero field for the shortrange model in $d=3$ at $T=0.20$ which is to be compared with the transition temperature of $T_{S G} \simeq 0.95$ [13] (from Katzgraber et al. [18]). The right hand figure is for the long-range model with $\sigma=0.75$, which is in the non mean-field, finite $T_{S G}$ region, see Fig. 5 . The temperature is $T=0.10$ which is to be compared with the mean field transition temperature of 1 , and the actual transition temperature of about 0.62. (From Katzgraber and APY [19].)

For the range of sizes that can be studied, which is quite large for the 1- $d$ model, we see that the data for $P(q)$ is consistent with the RSB picture and inconsistent with the droplet picture. Of course, it is possible that the sizes are too small to see the asymptotic critical behavior. However, if the droplet theory is correct asymptotically, one would need extremely large sizes to see it, especially for the 1- $d$ model.

\section{Ising Spin Glass in a Magnetic Field}

As discussed in Sec. 1, in the SK model there's a line of phase transitions in a magnetic field for an Ising spin glass, known as the Almeida Thouless [3] (AT) line, which separates a spin glass phase, with divergent relaxation times and "replica symmetry breaking", from a paramagnetic "replica symmetric" phase with finite relaxation times, see Fig. 2. Experimentally, it is harder to determine whether there is an AT line than whether there is a transition in zero field. The reason is that the experimentally measurable non-linear susceptibility, $\chi_{n l}$ diverges in zero field, providing a clear signatures of the transition, whereas $\chi_{n l}$ does not diverge on the AT line at non-zero field. Experiments therefore look for another signature of the transition, a divergent relaxation time. In a careful experiment [22], no such divergence in a field was found, implying the absence of an AT line. However, not all experiments have come to the same conclusion.

Although there is no static divergent quantity measurable in experiments, there is such a quantity which is accessible in simulations, namely the spin glass susceptibility $\chi_{S G}$. The zero field definition of $\chi_{S G}$ given in Eq. (4) is is generalized in a field to

$$
\chi_{S G}(\mathbf{k})=\frac{1}{N} \sum_{i, j}\left[\left(\left\langle S_{i} S_{j}\right\rangle-\left\langle S_{i}\right\rangle\left\langle S_{j}\right\rangle\right)^{2}\right]_{\mathrm{av}} e^{i \mathbf{k} \cdot\left(\mathbf{R}_{i}-\mathbf{R}_{j}\right)} .
$$

Equation (10) is just the "replicon mode" of replica field theory, which diverges for the SK model along the AT line. More conveniently, one can obtain from the simulations a correlation length 


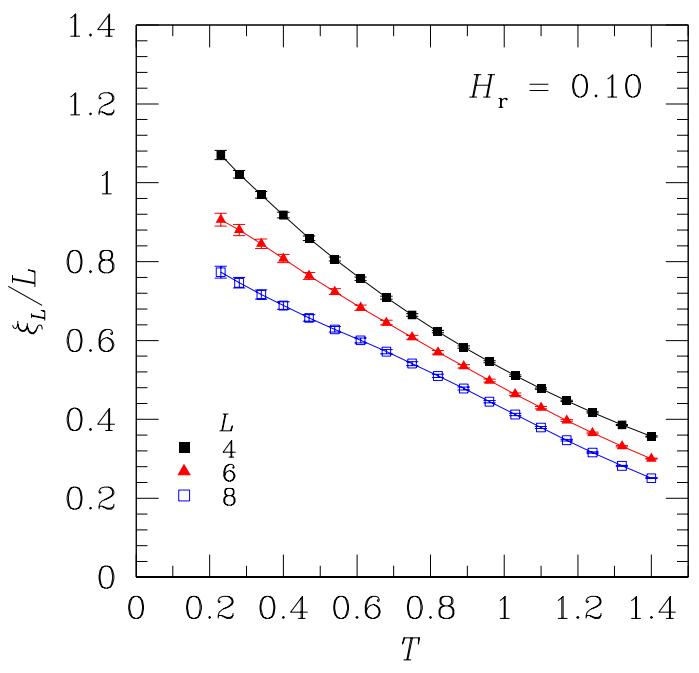

Figure 7. The scaled correlation length for the Ising spin glass with (random) field strength $H_{r}=0.1$ (H. G. Katzgraber and A. P. Young, unpublished).

$\xi_{L}$ from $\chi_{S G}(\mathbf{k})$ using Eq. (5), and this can be analyzed by FSS according to Eq. (7), so the transition is indicated by intersection of the data for different sizes.

In the simulations [24] we actually used a Gaussian random field, rather than a uniform field, for technical reasons, but MFT predicts an AT line for this case too, just as for a uniform field. Some results are shown in Fig. 7 for the three-dimensional, nearest-neighbor model. The strength of the (random) field is $H_{r}=0.1$ which is very small compared with the zero field transition temperature [13] $T_{S G} \simeq 0.96$. There is no intersection, which indicates that there is no AT line.

Subsequently, we [23] looked for the AT line in the 1-d model with long range interactions described in Sec. 4, and some data is shown in Fig. 8. One sees a clear intersection, indicating a transition in a field, i.e. an AT line, for $\sigma=0.55$, which is in the mean field regime. For $\sigma=0.75$ and 0.85 , which are in the non mean-field region, no AT line is seen. For the other value of $\sigma$ presented, 0.65 , which is close to the borderline value $(2 / 3)$ between the mean-field and non mean-field regions, there are intersections at low- $T$ for the smaller sizes, but the data for $L=128$ and 256 merge at low- $T$ indicating marginal behavior. The data for $L=512$ appears slightly higher at the lowest $T$ which could be equilibrated, but the difference is only about the same as the error bars.

Hence the data are consistent with there being an AT line in the region where the zero field transition is mean field-like. For the short range case this would be $d>6$. (I emphasize that we are not able to simulate directly the spin glass in a field for $d>6$, and this conclusion is obtained by analogy with the 1-d model with long-range interactions.)

\section{Conclusions}

The question we posed in this talk is whether the Sherrington-Kirkpatrick model is relevant for real spin glasses. Taken literally, the numerical results indicate that for $d>6$ the answer is "yes" while for $3 \leq d<6$ the answer is "only partly". For the latter case, the data for $P(q)$ are consistent with the RSB picture (i.e. with the behavior of the SK model) while the absence of an AT line disagrees with RSB picture. The numerical data for $d<6$ thus favor an intermediate scenario, called "TNT" by Krzakala and Martin [25], see also Ref. [26]. 

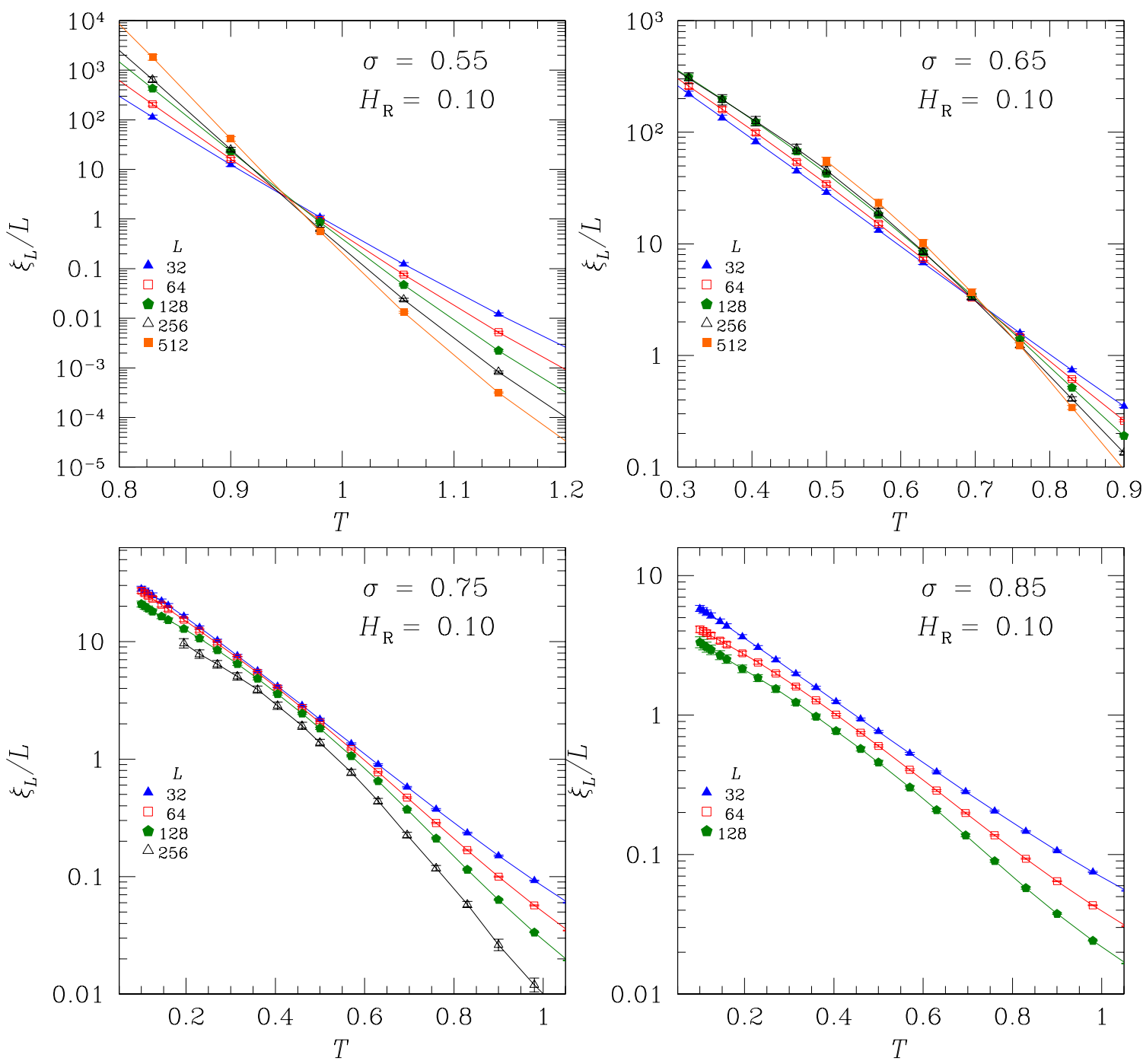

Figure 8. Data for the scaled correlation length of the one-dimensional model with long-range interactions in a magnetic field from Ref. [23]. Results are presented for $\sigma=0.55$, which is in the mean field regime (for the zero field transition), see Fig. 5, $\sigma=0.65$, which is close to the borderline between the mean-field and non mean-field regions, and $\sigma=0.75$ and 0.85 , which are in the non mean-field region. One sees an intersection, indicating a transition in a field, i.e. an AT line, only for $\sigma=0,55$. The case of $\sigma=0.65$ seems to be borderline, as discussed in the text.

However, it is possible that the numerical data are not in the asymptotic regime, and the devotees are the droplet picture claim that it does hold asymptotically (at least for $d<6$ ). It this is so, then it must only apply for quite large lattice sizes, (especially for the analogous one-dimensional model).

\section{Acknowledgments}

I acknowledge support from the National Science Foundation under grant DMR 0337049. I am also very grateful to the Hierarchical Systems Research Foundation for a generous allocation of computer time on its Mac G5 cluster. I thank my collaborators in the work described here, 
Helmut Katzgraber and Matteo Palassini, for many stimulating interactions.

\section{References}

[1] Edwards S F and Anderson P W 1975 J. Phys. F 5965

[2] Sherrington D and Kirkpatrick S 1975 Phys. Rev. Lett. 351792

[3] de Almeida J R L and Thouless D J 1978 J. Phys. A 11983

[4] Parisi G 1979 Phys. Rev. Lett. 431754

[5] Parisi G 1980 J. Phys. A. 131101

[6] Talagrand M 2006 Annals of Mathematics 163221

[7] Fisher D S and Huse D A 1986 Phys. Rev. Lett. 561601

[8] Huse D A and Fisher D S 1987 J. Phys. A 20 L997

[9] Fisher D S and Huse D A 1988 Phys. Rev. B 38386

[10] Bray A J and Moore M A 1986 Heidelberg Colloquium on Glassy Dynamics and Optimization ed Van Hemmen L and Morgenstern I (Springer) p 121

[11] McMillan W L 1984 J. Phys. A 173179

[12] Ballesteros H G, Cruz A, Fernandez L A, Martin-Mayor V, Pech J, Ruiz-Lorenzo J J, Tarancon A, Tellez P, Ullod C L and Ungil C 2000 Phys. Rev. B 6214237 (Preprint (arXiv:cond-mat/0006211))

[13] Katzgraber H G, Körner M and Young A P 2006 Phys. Rev. B 73224432 (Preprint (arXiv: cond-mat/0602212))

[14] Hukushima K and Nemoto K 1996 J. Phys. Soc. Japan 651604

[15] Boettcher S 2005 Phys. Rev. Lett. 95197205

[16] Fisher M E, Ma S k and Nickel B G 1972 Phys. Rev. Lett. 29 917-920

[17] Kotliar G, Anderson P W and Stein D L 1983 Phys. Rev. B 27602

[18] Katzgraber H G, Palassini M and Young A P 2001 Phys. Rev. B 63 184422 (Preprint (arXiv: cond-mat/0108320))

[19] Katzgraber H G and Young A P 2003 Phys, Rev. B 67134410 (Preprint (arXiv:cond-mat/0210451))

[20] Hartmann A K 1999 Phys. Rev. E 5984

[21] Boettcher S 2004 European Physics Journal B 3883

[22] Mattsson J, Jonsson T, Nordblad P, Katori H A and Ito A 1995 Phys. Rev. Lett. 744305

[23] Katzgraber H G and Young A P 2005 Phys. Rev. B 72184416 (Preprint (arXiv:cond-mat/0407031))

[24] Young A P and Katzgraber H G 2004 Phys. Rev. Lett. 93207203 (Preprint (arXiv:cond-mat/0407031))

[25] Krzakala F and Martin O C 2000 Phys. Rev. Lett. 853013 (Preprint (arXiv:cond-mat/0002055))

[26] Palassini M and Young A P 2000 Phys. Rev. Lett. 853017 (Preprint (arXiv:cond-mat/0002134)) 